\title{
A Systematic Approach to Orbital CT Scans: Integrating the BALPINE Method into Ophthalmology Curriculum
}

\author{
Frederick D. Nelson, MD ${ }^{1}$ Donovan S. Reed, MD ${ }^{1}$ Grant A. Justin, MD ${ }^{1}$ Hakeem F. Hindi, MD ${ }^{2}$ \\ Halward M. J. Blegen IV, DO ${ }^{1}$ Tatiana S. Zanganeh, MD ${ }^{1}$ Aaron M. Betts, MD ${ }^{2}$ Brett W. Davies, MD \\ ${ }^{1}$ Department of Ophthalmology, San Antonio Uniformed Services \\ Health Education Consortium, San Antonio, Texas \\ 2 Department of Radiology, San Antonio Uniformed Services Health \\ Education Consortium, San Antonio, Texas \\ ${ }^{3}$ Department of Research, Brooke Army Medical Center, Ft. Sam \\ Houston, Texas \\ J Acad Ophthalmol 2020;12:e92-e95.

\begin{abstract}
Address for correspondence Frederick D. Nelson, MD, Department of Ophthalmology, San Antonio Uniformed Services Health Education Consortium, 1100 Wilford Hall Loop, Lackland AFB, San Antonio, TX 78236 (e-mail: Frederick.d.nelson12.mil@mail.mil).
\end{abstract}

\section{Abstract}

\section{Keywords}

- ophthalmology

- radiology

- computed tomography

- resident education

- orbit
Objective While multiple approaches to the evaluation of orbital computed tomography (CT) imaging have been proposed in both ophthalmology and radiology literature, there remains a paucity of standardized approaches to orbital CT interpretation education for the ophthalmologist in training. To address this, the bones, anterior chamber, lens, posterior globe, intraconal orbit, neurovascular structures, and extraocular muscles (BALPINE) method was selected and its utilization tested as an education tool to improve resident competency in the proper assessment of orbital CT imaging.

Methods The BALPINE method is an established systematic approach to reading orbital CT scans. This approach was selected as a standardized education tool after a systematic review of orbital CT evaluation methods in the ophthalmology and radiology literature. A 15-item test, comprised of frequently diagnosed orbital pathologies developed in conjunction with a radiologist, was designed to test a group of ophthalmologists in training across multiple residency programs. The in-training ophthalmologists were first given this test prior to being taught the BALPINE standardized method to assess orbital CT imaging. The study participants were then administered a lecture describing the BALPINE method approach to orbital CT imaging. The same study participants were then administered the same test to assess improvement. The lecture was developed and administered by a board-certified Oculoplastic surgeon. The lecturer was blinded to the images included in the examination during lecture development. The radiologist was blinded to the lecture and the BALPINE method; thus, the images selected were not specific to the method being taught.

Results Overall, 27 residents, interns, and medical students completed the prelecture test, BALPINE method curriculum lecture, and postlecture test. There was an average of a 2.3-point $(15 \%)$ significant improvement per test score $(p<0.0001)$. Each level of training demonstrated improvement pre- and posttest and overall scores improved with each additional year of training, indicating the test was an appropriate measurement of ability and understanding. received

April 6, 2020

accepted

May 18,2020
DOI https://doi.org/

$10.1055 / \mathrm{s}-0040-1713939$

ISSN $2475-4757$.
Copyright $\odot 2020$ by Thieme Medical

Publishers, Inc., 333 Seventh Avenue, New York, NY 10001, USA Tel: +1(212) 760-0888.
License terms

()ㅇ (1) $\Theta \circledast$ 
Conclusion Teaching a systematic approach to the evaluation of orbital CT scans improves an ophthalmologist in training's ability to diagnose orbital pathology efficiently and effectively. Widespread residency curriculum adoption of the BALPINE method would enhance the training ophthalmologist's diagnostic capability.

Orbital computed tomography (CT) has become the standard for diagnosing orbital injuries in the acute setting. Despite a decrease in contrast sensitivity compared with magnetic resonance imaging (MRI), ${ }^{1} \mathrm{CT}$ has remained the diagnostic imaging modality of choice due to efficiency, availability, and necessary sensitivity for diagnosis, particularly regarding bony anatomy. Quick decisions by ophthalmologists and ophthalmology residents are critical for appropriate management and treatment of acute orbital injuries. However, there is currently no standardized resident education curriculum regarding a systematic approach to evaluating orbital CT imaging.

Since 1985, efforts have been made through studies and publications to create a systematic approach for ophthalmologists to use when evaluating CT scans. ${ }^{2-6}$ One study showed that various CT findings could be used to predict treatment and surgical management of orbital injuries. ${ }^{7}$ Despite these efforts, no approach has been widely adopted by ophthalmologists or used to teach residents. Incorporating a systematic approach to the evaluation of CT scans in resident curriculum would be beneficial for competency of ophthalmologists.

The purpose of this study is to test the efficacy of implementing a simple systematic approach to the evaluation of orbital CT imaging within the resident education curriculum. Through a literature review, and in collaboration with oculoplastic and neuroradiology specialists, the bones, anterior chamber, lens, posterior globe, intraconal orbit, neurovascular structures, and extra ocular muscles (BALPINE) method was identified to be both simple and comprehensive. The BALPINE method was developed in 2014 to address the deficiency of a standardized approach to the evaluation of orbital CT scans. ${ }^{2}$ As an effective standardized approach to orbital CT imaging, the utility of implementing this methodology was tested on ophthalmologists in training across multiple institutions.

\section{Methods}

The BALPINE method, a systematic approach to reading orbital CT scans was selected after a systematic review of the literature was performed.

The systematic review began with database searches for articles. The databases selected were PubMed and CINAHL. Search terms were then selected, with the goal of being both comprehensive and specific to the topic under question. The years included in the search were 1980 to 2018. The search terms utilized were the following, with the amount of articles per database in parenthesis:
- Systematic approach AND orbital computed tomography (PubMed 30), (CINAHL 2).

- Reading orbital computed tomography (PubMed 10), (CINAHL 3).

- Orbital computed tomography and Systematic (PubMed 158), (CINAHL 2).

Specific inclusion and exclusion criteria were established for article extraction. Articles were included if they discussed a systematic approach to reading orbital CT scans. Articles were excluded if they focused evaluating on only one specific part of the orbital CTscan, were specific to one type of injury on orbital CT scans (i.e., orbital wall fractures), or did not discuss specifically how to approach evaluation of a CT scan (i.e., discussing anatomy of an orbital CT scan but not how to interpret it). These inclusion and exclusion criteria were used in determining which articles should be used in the final analysis.

The article extraction was performed in three phases. First, the title review resulted in 12 articles that were extracted. Next, an abstract review of the articles was completed to further identify pertinent articles. This step yielded eight articles for the review. Lastly, a full paper review of all eight of the selected articles was performed, which led to the selection of five articles to be utilized in the final review. ${ }^{2-6}$ of the orbital CT evaluation approaches, the BALPINE method was determined to be the simplest to implement, while still being comprehensive. ${ }^{7,8}$

To test the effectiveness of the CT evaluation method, an examination composed of CT orbital images was developed with the aid of the Ophthalmology and Radiology departments at San Antonio Uniformed Services Health Education Consortium (SAUSHEC). The examination assessed the ability to recognize and diagnose orbital pathology and trauma. This test consisted of 15 images. A lecture was also designed by an oculoplastic surgeon (B.W.D) on how to implement the BALPINE method. The lecture developed was roughly 15minute long. The lecturer was blinded to the images and pathology included in the examination. The radiologist (H.F. $\mathrm{H}$ ) who obtained the images for the examination was also blinded to the BALPINE method and the lecture, only receiving instruction to provide images with orbital pathology obtained from orbital CTs, with an emphasis on traumatic diagnoses. Thus, the images were selected independent of the instruction process. ${ }^{9-11}$

The diagnoses included in the examination were as follows: corneal foreign body, orbital cellulitis with subperiosteal abscess, orbital lymphoma, ruptured globe, dacryocytsitis, zygomaticomaxillary complex fracture, preseptal and orbital cellulitis, ruptured globe, idiopathic orbital inflammation, 
medial wall fracture with medial rectus herniation, lens dislocation, dilated superior ophthalmic vein from carotidocavernous fistula, adenoid cystic carcinoma of the lacrimal gland, penetrating left globe injury with intraocular foreign body, and thyroid eye disease. ${ }^{7}$

The test was administered in a pre- and postlecture format. The 15-image examination was given to 27 trainees; medical students (seven), postgraduate year level 1 (PGY1; four), postgraduate year level 2 (PGY2; eight), postgraduate year level 3 (PGY3; five), and postgraduate year level 4 (PGY4; three). The examination was administered before and after the BALPINE lecture and scores compared. The examinees came from two separate ophthalmology programs. The two residency programs that were included were the SAUSHEC Ophthalmology and the University of Texas Health Science Center of San Antonio (UTHSCSA) Ophthalmology programs. The format of the examination answers was free text, to more closely simulate real world diagnosis. The results were then scored and analyzed.

\section{Results}

Overall, 27 residents, interns, and medical students completed the prelecture test, lecture, and postlecture test. There was an average of a 2.3-point (15.33\%) improvement per test score $(p<0.0001)$. The results demonstrate that with appropriate instruction and utilization of the BALPINE method, there is a statistically significant improvement in ability to recognize and diagnose pathology based on orbital CT imaging. ${ }^{8}$

In a subgroup analysis, examinees were separated by year of training with groups being medical students, PGY1, PGY2, PGY3, and PGY4. Overall there was a trend of improvement in test scores, pre- and posttest per level of training as indicated in -Table 1. This indicates that the test was an appropriate tool for assessing knowledge, as it would be expected that with increased training, scores should also increase. Additionally, among examinees, no individual had a worse posttest score than the pretest; however, three test takers showed no improvement. Of the 27 test takers, 24 (89\%) showed an improvement in the posttest score, with an average improvement of 2.3 points.

Table 1 Mean pretest and posttest scores with the average change for each year of training, as well as overall mean values and $p$-value

\begin{tabular}{|l|l|l|l|}
\hline Training level & $\begin{array}{l}\text { Mean } \\
\text { pretest }\end{array}$ & $\begin{array}{l}\text { Mean } \\
\text { posttest }\end{array}$ & $\begin{array}{l}\text { Average } \\
\text { change }\end{array}$ \\
\hline Medical students & 3.57 & 5.14 & 1.57 \\
\hline PGY1 & 4.50 & 7.75 & 3.25 \\
\hline PGY2 & 6.13 & 8.25 & 2.13 \\
\hline PGY3 & 7.40 & 9.40 & 2.00 \\
\hline PGY4 & 6.33 & 10.0 & 3.67 \\
\hline Overall & 5.48 & 7.78 & 2.29 \\
\hline
\end{tabular}

$p$-Value $<0.0001$

Abbreviation: PGY, postgraduate year.

\section{Discussion}

Overall, the results of this study demonstrate that receiving instruction on orbital CT evaluation with the BALPINE method enhances trainee's ability to effectively evaluate orbital CT imaging. As above, $89 \%$ showed an improvement in the posttest score, with an average improvement of 2.3 points. This result was significant, indicating that receiving instruction on and utilizing the BALPINE method indeed improves ability to read and diagnose pathology on an orbit CT. Additionally, 0\% of examinees showed worsening on the posttest. This result points to the simplicity of application of the BALPINE method, as it did not confuse examinees nor cause them to question the existing diagnoses they had previously identified.

Interestingly, the medical student and PGY1 subgroups, while showing overall improvement in their posttest scores (average improvement of 1.57 and 3.25), did not reach statistical significance, while PGY2, PGY3, and PGY4 all showed significant improvement in their posttest scores. While sample size may have contributed, this finding may indicate that such a lecture and test requires a baseline understanding of the orbit and orbital anatomy for the BALPINE method to be the most effective. While it is clear that students with lower training will still benefit, maximum benefit is likely achieved when delivered to those with a certain level of understanding of orbital anatomy and pathology.

\section{Limitations}

Limitations of the study include the relatively small sample size. However, despite the small sample size, the improvement demonstrated remained significant. Another limitation of the study is the lack of multiple slices or cuts of the CT images shown during examination. The lack of a series of images may make appropriate diagnosis from orbital CT imaging more difficult. If each test image was administered in a scroll through window, diagnosis would likely have been more accurate, which may have made the data more reliable. Ultimately, a single image approach was chosen for ease of administration. Nevertheless, with the pre- and posttest format, it still provided a reliable test for evaluating improvement and accuracy, which was ultimately the purpose of the study. While students may have had improved overall scores with a scroll through method, they were still be able to demonstrate diagnostic improvement with single images following implementation of the BALPINE method.

\section{Conclusion}

Implementation of a standardized approach to orbital CT imaging utilizing the BALPINE method improves ability of an ophthalmologist in training to evaluate and diagnose orbital pathology on orbital CT imaging. There remains a lack of standardized instruction on orbital imaging in the ophthalmology resident education curriculum. The BALPINE method, which is a simple and comprehensive systematic 
approach to the evaluation of orbital CT imaging, has been demonstrated to significantly improve the diagnostic abilities of ophthalmologists in training.

The incorporation of this standardized method to orbital CT imaging within the resident education curriculum has the potential to greatly impact not only the training ophthalmologist but also patient care. Future directions include testing the BALPINE method at additional sites to obtain a greater sample size to confirm the initial results of this study. Ultimately, the results of this study suggest widespread adoption of the BALPINE method in ophthalmology residency curriculum would likely enhance the training ophthalmologist's diagnostic ability and ultimately, their ability to care for patients.

\section{Note}

The view(s) expressed herein are those of the author(s) and do not reflect the official policy or position of Brooke Army Medical Center, the U.S. Army Medical Department, the U.S. Army Office of the Surgeon General, the Department of the Air Force, the Department of the Army, Department of Defense, the Uniformed Services University of the Health Sciences or any other agency of the U.S. Government.

Funding

None.

Conflict of Interest

None declared.

\section{Acknowledgment}

The authors thank James K. Aden, PhD.

\section{References}

1 Lee H-J, Jilani M, Frohman L, Baker S. CT of orbital trauma. Emerg Radiol 2004;10(04):168-172

2 Betts AM, O'Brien WT, Davies BW, Youssef OH. A systematic approach to CT evaluation of orbital trauma. Emerg Radiol 2014;21(05):511-531

3 Naik MN, Tourani KL, Sekhar GC, Honavar SG. Interpretation of computed tomography imaging of the eye and orbit. A systematic approach. Indian J Ophthalmol 2002;50(04):339-353

4 Simon GBb, Annunziata CC, Fink J, Villablanca P, McCann JD, Goldberg RA. Rethinking orbital imaging: establishing guidelines for interpreting orbital imaging studies and evaluating their predictive value in patients with orbital tumors. Ophthalmol 2005;112(12):2196-2207

5 Cobb SR, Yeakley JW, Lee KF, Mehringer CM, Grinnell VS. Computed tomographic evaluation of ocular trauma. Comput Radiol 1985;9(01):1-10

6 Nguyen VD, Singh AK, Altmeyer WB, Tantiwongkosi B. Demystifying orbital emergencies: a pictorial review. Radiographics 2017; 37(03):947-962

7 Schouman T, Courvoisier DS, Van Issum C, Terzic A, Scolozzi P. Can systematic computed tomographic scan assessment predict treatment decision in pure orbital floor blowout fractures? J Oral Maxillofac Surg 2012;70(07):1627-1632

8 Tawfik HA, Abdelhalim A, Elkafrawy MH. Computed tomography of the orbit - A review and an update. Saudi J Ophthalmol 2012;26 (04):409-418

9 Kuhn F, Morris R, Witherspoon CD, Mann L. Epidemiology of blinding trauma in the United States Eye Injury Registry. Ophthalmic Epidemiol 2006;13(03):209-216

10 Guly CM, Guly HR, Bouamra O, Gray RH, Lecky FE. Ocular injuries in patients with major trauma. Emerg Med J 2006;23(12): 915-917

11 Scruggs D, Scruggs R, Stukenborg G, Netland PA, Calland JF. Ocular injuries in trauma patients: an analysis of 28,340 trauma admissions in the 2003-2007 National Trauma Data Bank National Sample Program. J Trauma Acute Care Surg 2012;73(05): $1308-1312$ 